\title{
Simulation of Partial Discharge Induced Electromagnetic Wave in Power Transformer
}

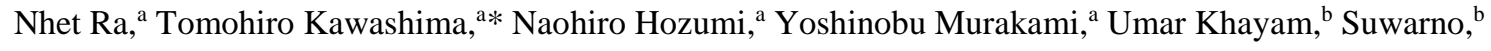 \\ ${ }^{a}$ Departement of Electrical and Electronic Information Engineering, Toyohashi University of Technology, Email: n169202@edu.tut.ac.jp

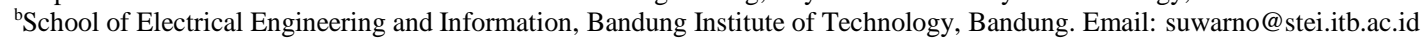

\begin{abstract}
This paper describes the numerical simulation of partial discharge (PD) in oil-filled power transformer using FDTD method (Finite Difference Time Domain). We investigate the effects of oil insulation medium and internal structures on the propagation of electromagnetic signal emitted from the partial discharge source. In this study, partial discharge is modeled as the Gaussian Pulse (pulse's width 1 ns). To observe the electromagnetic intensity and also the voltage distribution, three sensors are employed. The magnitude, frequency, and attenuation of the EM wave without internal structures and with internal structures are compared. The Results show that the EM wave intensity is attenuated while propagating through the oil insulation, iron-core, and windings. And the time arrival of each signal is different due to its distance from the PD source. The simulation results in both cases show that the first dominance frequency occurs in $1 \mathrm{GHz}$ of frequency. However, the frequency spectrum of simulation with internal structures has resonance frequencies in the low region from $80 \mathrm{MHz}$ to $300 \mathrm{MHz}$ of frequency. These resonance frequencies occur because the windings are considered to be a loop antenna.
\end{abstract}

Keywords: Electromagnetic wave (EMW); FDTD approach; Ultra High Frequency (UHF)

\section{Introduction}

Power transformer is one of the most important elements in power transmission and distribution systems. During the operation, the threat such as lightning strikes, switching transient, and short-circuit, can lead to the immediate failure, especially for the aged transformer. When the insulation is degraded, it cannot withstand such events. The insulation degradation of partial discharge is frequently linked to partial discharge [1,2].

The occurrence of partial discharge is accompanied by some energy such as impulse current, heat light radiation, electromagnetic wave, and mechanical wave [3, 4]. Various methods have been proposed for detecting partial discharge by mean of the energy released from partial discharge. The convention method, specified in the IEC 60270 standard, is extensively used to detect the impulse current induced by partial discharge [5]. The integration of the discharge current over time through the detecting impedance is the apparent charge corresponding to the partial discharge. The

${ }^{*}$ Corresponding author. Tel.: +81-903-425-481

Address:1-1 Hibarigaoka, Tempaku

Toyohashi, Aichi, JAPAN 441-8580 insulation assessment can also be done by chemical method. A chemical test such as High-Performance Liquid Chromatography (HPLC) and Dissolved Gas Analysis (DGA) are employed to detect the gasses and components produced due to the breakdown of voids. Since partial discharge accompanied by an acoustic wave and an electromagnetic wave, a newly developed sensor such as an acoustic sensor, and UHF sensor are usually been employed for PD detection and finding the location of the defects [612].

However, it is very difficult for UHF antenna to locate the defects when partial discharge occurs and propagates through complex structures and the insulation medium of the transformer [13-15]. Therefore, it is necessary to examine the effects of transformer components such as windings, pressboard and iron core on EM wave propagation. In addition, the reliability enhancement of the diagnosis, such as PD location identification and increase the detection sensitivity, it is strongly required to understand the propagation properties of the EM waves induced by PD occurring inside the transformer.

From this point of view, we proposed to investigate the insulation diagnostics technique for oil-filled transformer using the UHF methods through numerical simulation using finite difference time domain (FDTD) method. 


\section{Finite Difference Time Domain Method (FDTD)}

\subsection{FDTD Formula}

The finite difference time domain method (FDTD) has been widely used for solving electromagnetic filed problems [16]. This technique was proposed by Kane Yee in 1996, specifically for solving Maxwell's equation and curl equation.

The idea of solving the electromagnetic field using Finite Difference Time Domain (FDTD) is a leapfrog manner in which the electric field is computed for given instant time, then the magnetic field is obtained in the next instant time, and the process is repeated over and over again. The finite difference technique is based on approximations which permit replacing differential equations by finite difference equations. These finite difference approximations are algebraic in form and they relate the value of the dependent variable at a point in the solution region to the values at some neighboring points. The basic steps involved are:

- Dividing the solution region into a grid of nodes: The commonly used grid patterns are rectangular, skew, triangular and circular grid

- Approximating the given differential equation by its finite difference equivalent that relates the dependent variable at a point in the solution region to its values at the neighboring points

- Solving the difference equations subject to the prescribed boundary and initial conditions.

\subsection{Yee's FDTD algorithm}

Maxwell's equations in an isotropic medium [16] are:

$$
\nabla \times \vec{E}=-\mu \frac{\partial \vec{H}}{\partial t}
$$

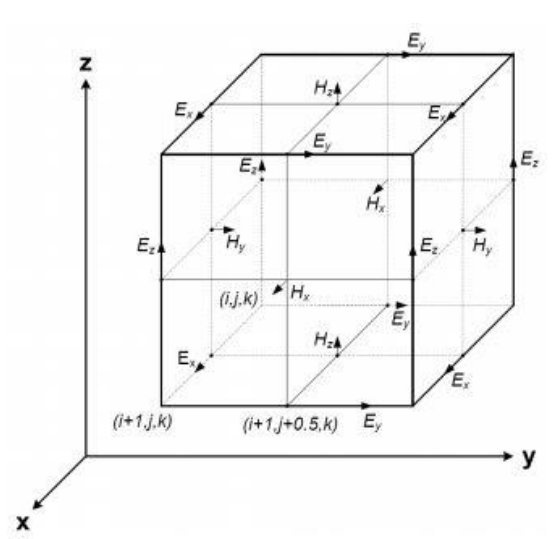

Figure 1. Yee's grids for finite difference time domain. The electric ' $E$ ' and magnetic field ' $H$ ' [16]

$$
\nabla \times \vec{H}=\sigma \vec{E}+\varepsilon \frac{\partial \vec{E}}{\partial t}
$$

where ' $E$ ' is the electric field (v/m), ' $H$ ' is the magnetic field $(\mathrm{A} / \mathrm{m})$, ' $\varepsilon$ ' is dielectric constant $(\mathrm{F} / \mathrm{m})$, ' $\mu$ ' is the permeability $(\mathrm{H} / \mathrm{m})$ and ' $\sigma$ ' is the conductivity $(\mathrm{S} / \mathrm{m})$ of the medium. Yee's defined the object into small grids and the grid points in the solution region needed to be defined as a function of space and time. The electric ' $E$ ' and magnetic field ' $H$ ' of a unit cell is shown in Fig. 1.

\subsection{Absorbing Boundary condition}

Absorbing boundary conditions known as ABCs method has been widely used for the boundary condition. Absorbing boundary condition allows an ongoing electric field ' $E$ ' and magnetic field ' $H$ ' without reflected back to the problem space. The most efficient of $\mathrm{ABCs}$ circuit is a perfect matched layer (PML) [17]. If a wave is propagating in medium $\mathrm{A}$ and it strikes upon medium $\mathrm{B}$, the amount of reflection is dictated by the intrinsic impedances of the two media, which is given by:

$$
\Gamma=\frac{\eta_{A}-\eta_{B}}{\eta_{A}+\eta_{B}}
$$

The impedances are determined by " $\varepsilon$ " and " $\mu$ " of the two media:

$$
\eta=\sqrt{\frac{\mu}{\varepsilon}}
$$

\subsection{PD Pulse Modeling}

A typical pulse can be numerically simulated by a Gaussian function as [18]:

$$
i(t)=I_{0} \exp \left[\frac{-\left(t-t_{0}\right)^{2}}{2 \sigma^{2}}\right]
$$

where $I_{0}$ is the amplitude to the time instant measured at the center of the pulse, $\sigma$ is the characteristic waveform parameter which describes the current pulse width at half the maximum value, which for a PD pulse is equal to $2.36 \sigma$.

Table 1 shows the impulse current's properties of the PD pulse model. Fig. 3 shows the model of impulse current corresponded to the partial discharge. Fig. 4 shows the model of inverse fast furrier transforms (IFFT) of impulse current.

Table 1. Impulse current's propagation

\begin{tabular}{lll}
\hline Parameters & Formula & Value \\
\hline Sigma & $=(4 \times f m a x)^{2}$ & $1.6 \times 10^{19}$ \\
Time & $\mathrm{T}_{0}(\mathrm{~s})$ & $0(\mathrm{~s})$ \\
& $\mathrm{T}_{\mathrm{e}}(\mathrm{s})$ & $20 \times 10^{-9}(\mathrm{~s})$ \\
\hline
\end{tabular}




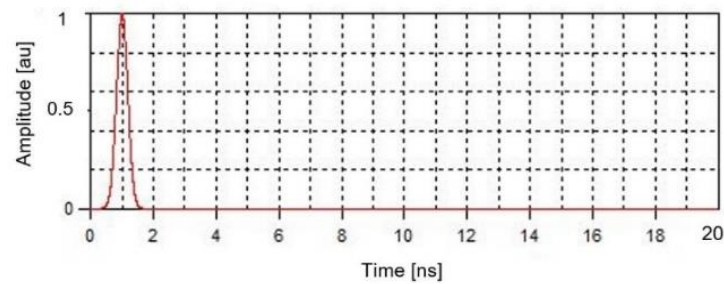

Figure 2. Model of impulse current corresponded to the partial discharge.

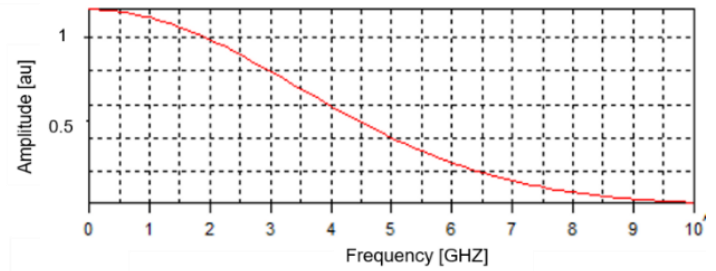

Figure 3. Model of inverse fast furrier transform IFFT of impulse current

\section{Simulation Model}

Figure 4 shows the cut-view of the simplified three-phase transformer using Magna TDM (CTC: Magna version 8.0). The model consists of transformer tank, iron core, windings, and oil insulation medium. The dimension of the transformer tank is $2000 \mathrm{~mm} \times 4000 \mathrm{~mm} \times 2400 \mathrm{~mm}$. The model has 3 windings with the $400 \mathrm{~mm}$ outer radius and $200 \mathrm{~mm}$ inner radius each. These windings were wrapped around the iron.

In this study, we placed a partial discharge source (PD) on the surface of the transformer windings. The electromagnetic wave induced from the discharge point is propagated past through the oil insulation and internal structures of the power transformer. Finally, the electric field due to the partial discharge is observed at three different points.

The finite difference time domain (FDTD) is used to compute the electric field and voltage distribution due to the partial discharge. The mesh $\mathrm{x}, \mathrm{y}, \mathrm{z}$ sized was selected as 2 $\mathrm{mm}, 2 \mathrm{~mm}, 2 \mathrm{~mm}$, respective. The simulation was performed without internal structures and with internal structures filled with oil insulation medium (the oil relativity permittivity $\varepsilon \mathrm{r}=2.2$ ).

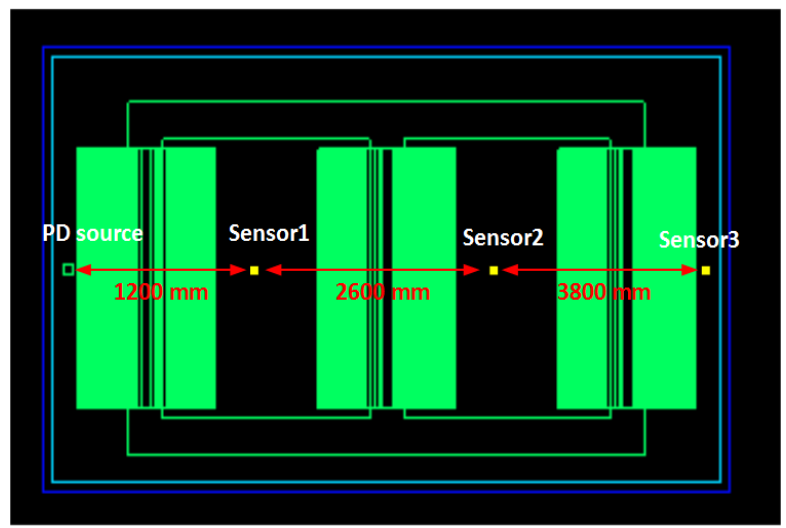

Figure 4. The model of a transformer with internal structures (oil insulation, core, and windings) for FDTD simulation

\section{Simulation Results}

The electric field intensity and voltage distribution in the $\mathrm{x}, \mathrm{y}, \mathrm{z}$ directions due to the partial discharge were detected. The results from this study is separated into two sections, simulation without internal structures and with internal structures as follow:

\subsection{Electric Field intensity}

- Simulation results without internal structures

Figures 5, 6 and 7 show the $\mathrm{x}, \mathrm{y}, \mathrm{z}$ component of the electric field intensity of the electric field of sensor 1 , sensor 2 , and sensor 3 without internal structures. The electric field intensity in the $\mathrm{x}$-direction and $\mathrm{z}$-direction are slightly different in magnitude. However, in the y-direction the magnitude is high due the $\mathrm{y}$ direction is used as the propagation of EMW. The electric field intensity detected by sensor 1 is higher compared to sensor 2 and 3.

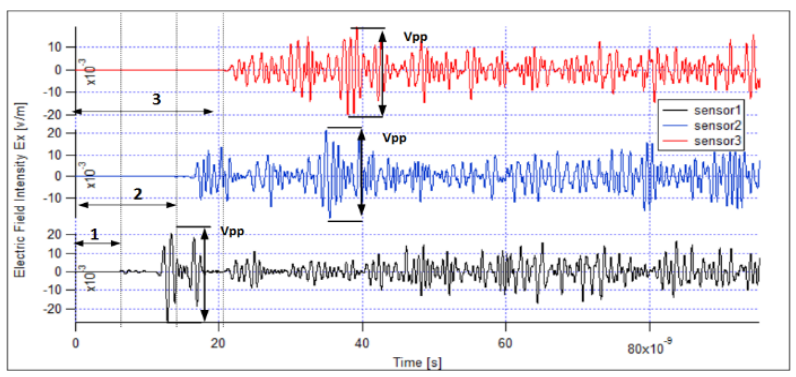

Figure 5. Simulation result of EMW in x-direction of power transformer without internal structure

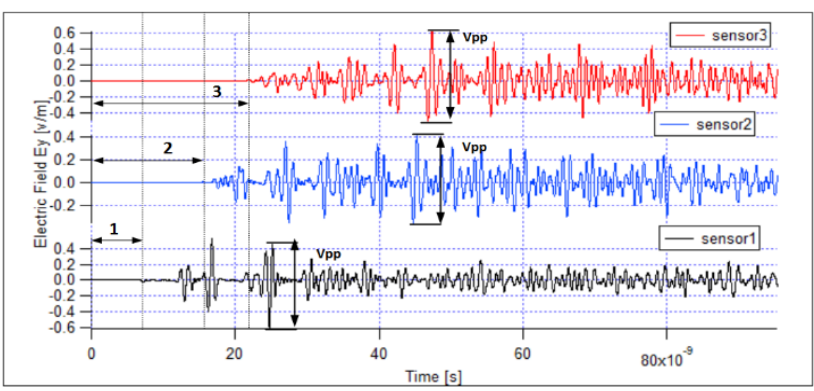

Figure 6. Simulation result of EMW in y-direction of power transformer without internal structure

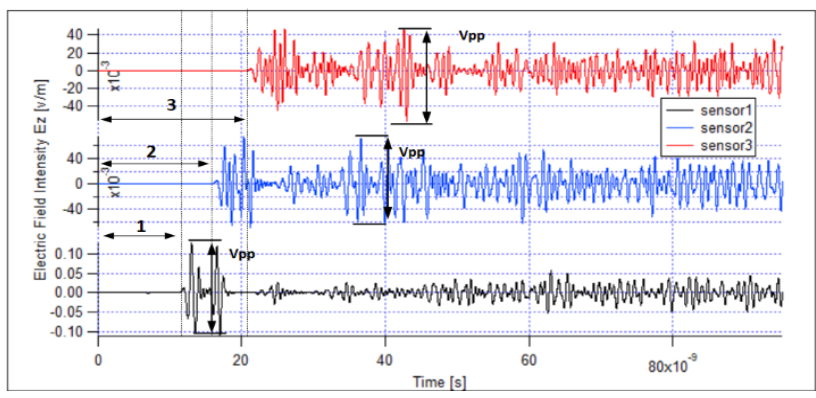

Figure 7. Simulation result of EMW in z-direction of power transformer without internal structure 
In addition, the signal obtained from sensor 1 is shorter than sensor 2 and sensor 2 is shorter sensor 3 respectively.

- Simulation Results With internal structures

Figures 8, 9 and 10 show the $\mathrm{x}, \mathrm{y}, \mathrm{z}$ component of the electric field intensity of the electric field of sensor 1 , sensor 2 , and sensor 3 with internal structures. The peak to peak point is delayed due to the internal structures of the transformer. The electric field intensity in the $\mathrm{x}$-direction and $\mathrm{z}$-direction are slightly different in magnitude. However, on the $y$-direction the magnitude is high due the $y$ direction is used as the propagation of EMW. The electric filed intensity detected by sensor 1 is higher compared to sensor 2 , and 3 . In addition, the signal derived from sensor 1 is shorter than sensor 2 and sensor 2 is shorter sensor 3 respectively.

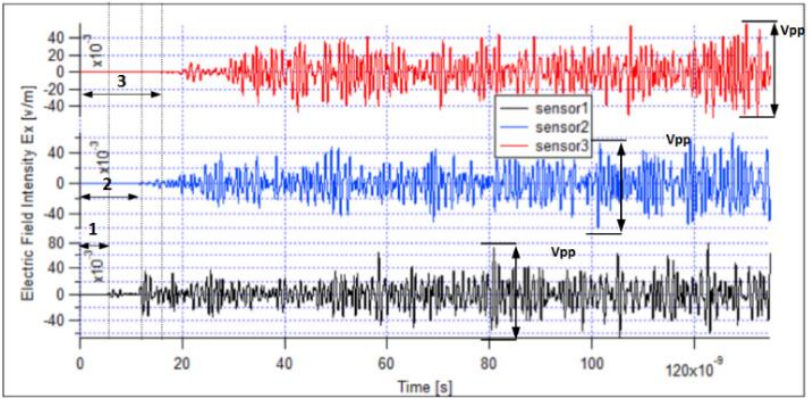

Figure 8. Simulation results of EMW in x-direction of power transformer with internal structure

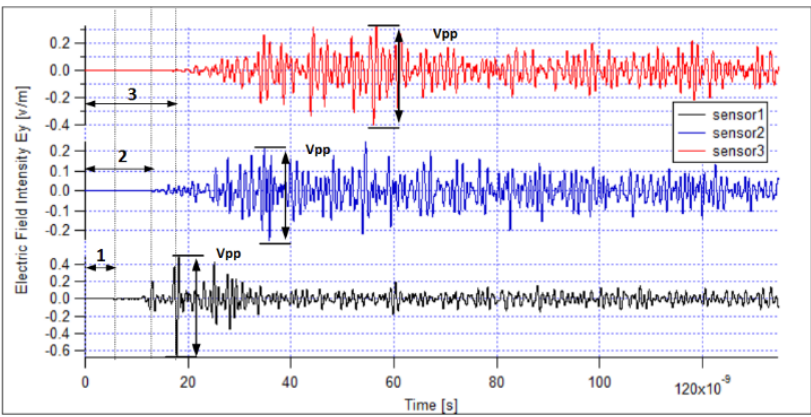

Figure 9. Simulation result of EMW in y-direction of power transformer with windings and core

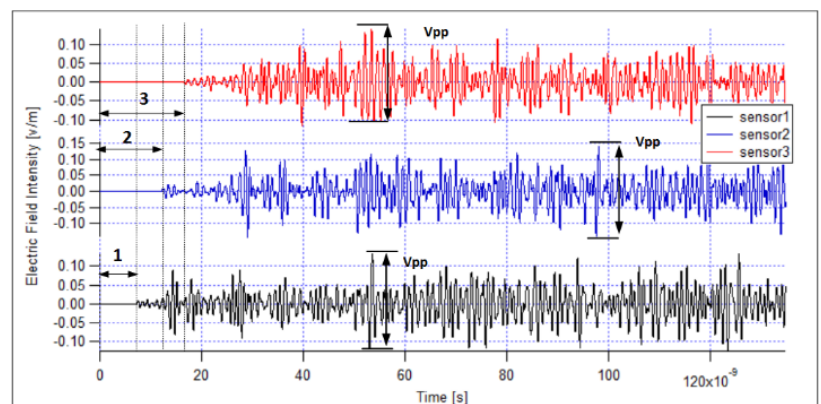

Figure 10. Simulation result of EMW in z-direction of power transformer with internal structure

\subsection{Voltage Distribution}

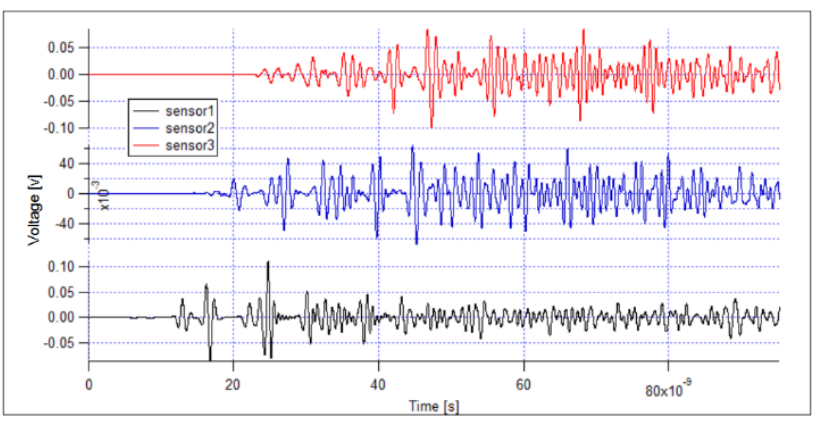

Figure 11. Simulation result of voltage distribution of power transformer without internal structures

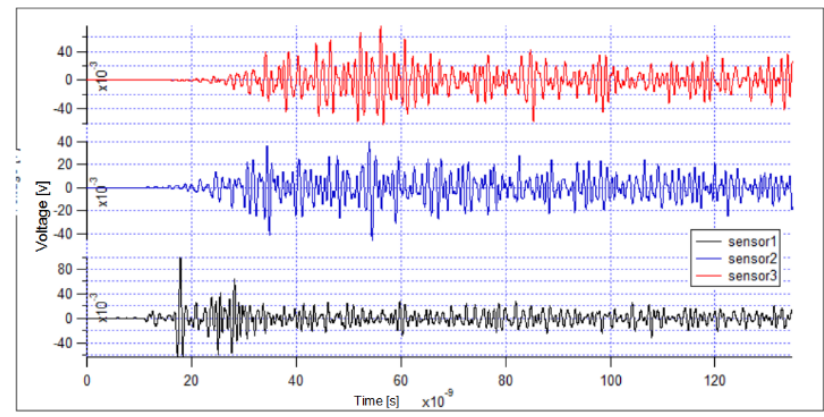

Figure 12. Simulation result of voltage distribution of power transformer with internal structures

Figures 11 and 12 shows the voltage distribution obtained from PD source, sensor 1, 2, and 3 with the peak amplitude $-1,0.09,-0.05,0.07 \mathrm{~V}$ respectively. The voltages derived from each sensor are slightly different in magnitude. However, the time difference to detect the PD signal is different.

\section{Analysis and Discussion}

It is evident from Figs. 13, 14 and 15 that frequency components around $1 \mathrm{GHz}$ have maximal and the resonance distinctly occurs in the low-frequency region at 80, 160 and $240 \mathrm{MHz}$ The resonance frequencies can be explained as follows.

The resonance is calculated by the existence of the windings. Namely, the windings work as a loop antenna. In the simulation model, the windings radius is $=0.4 \mathrm{~m}$. When the windings are considered to be a loop-antenna, the resonance frequency $f_{r}$, is calculated using Eq. 6, respectively.

$$
f_{r}=\frac{3.0 \times 10^{8}}{2 \pi r \sqrt{\varepsilon_{r}}}
$$

The resonance frequency of $80.47 \mathrm{MHz}$ derived from equation agrees well with that of $80 \mathrm{MHz}$ obtained from the FDTD simulation. Accordingly, it is indicated that the frequencies $160,240 \mathrm{MHz}$ and so on are attributed to the 
secondary and ternary resonance frequency of the windings looked like a loop antenna.

Moreover, the FFT spectrum without the windings exhibits no such resonance peaks in the low-frequency region, while it has a similar spectrum intensity around 1 $\mathrm{GHz}$ of the high-frequency region compared with the result without the windings.

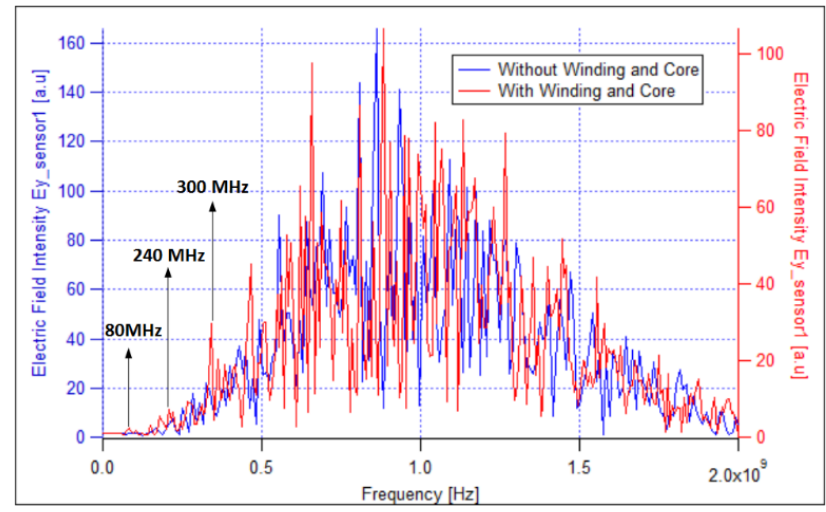

Figure 13. Simulation result of EMW frequency spectrum of power transformer detected by sensor 1

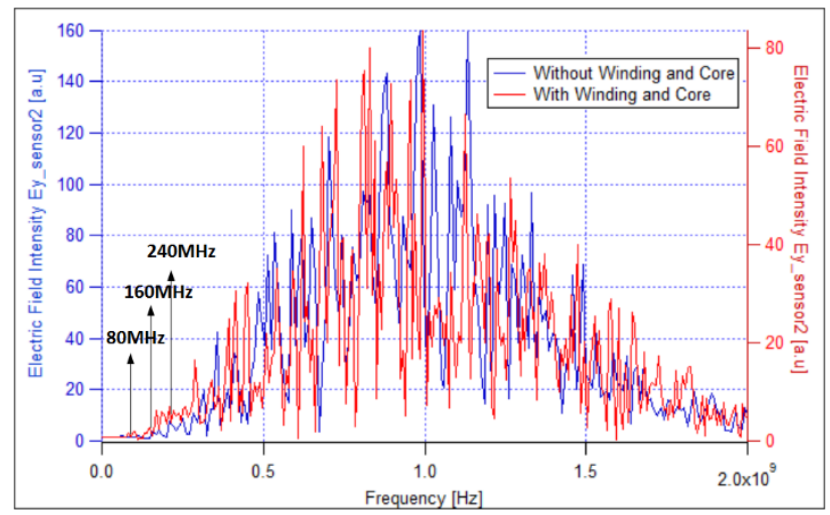

Figure 14. Simulation result of EMW frequency spectrum of power transformer by sensor 2

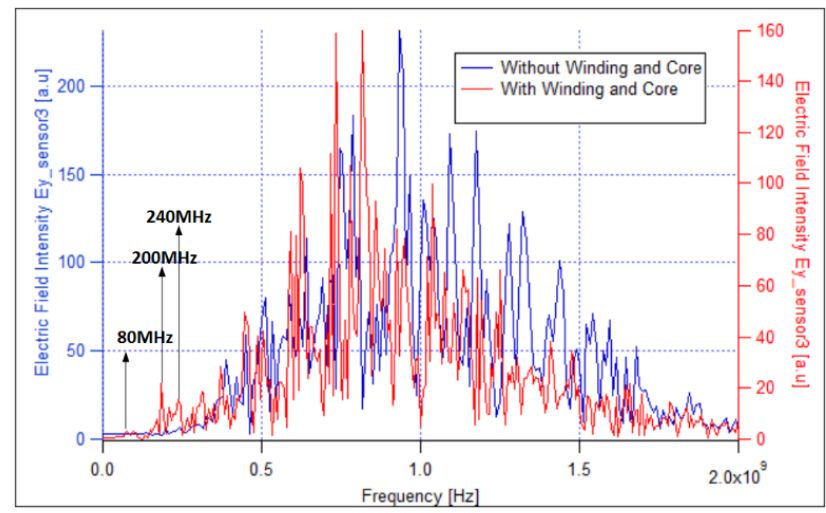

Figure 15. Simulation result of EMW frequency spectrum of power transformer detected by sensor 3

\section{Conclusion}

We investigated the effects of oil insulation medium and internal structures on the propagation of electromagnetic signal emitted from the partial discharge source. The Result showed that the EM wave intensity attenuated while propagating through the oil insulation, iron-core, and windings. And the time arrival of each signal was different due to its distances from the PD source. The first dominance frequency of the simulation without internal structures and with internal structures (oil insulation, windings, and core) occurred in $1 \mathrm{GHz}$ of frequency. However, frequency spectrum of simulation with internal structures (oil insulation, windings, and core) had a resonance frequency in the low region from $80 \mathrm{MHz}$ to $300 \mathrm{MHz}$ of frequency. This resonance frequencies occurred because the windings of the transformer acted as a loop antenna.

\section{Acknowledgements}

The authors would like to acknowledge to AUNSEED/Net Program for their funding support to make this research done.

\section{References}

[1] S. V. Kulkarni., S.A. Khapade, “ Transformer Engineering Design and practice". Indian Institute of Technology, Bombay Mumbai, India, 2004, pp. 389-409.

[2] Suwarno, "Partial Discharge in high voltage insulations" in High Voltage diagnosis, Bandung, penerbit, 2010, pp.4-39.

[3] H, Masayuki, K. Masahiro, "Recent Progress in Diagnosis of Transformers using Non-Conventional Partial Discharge Measurements," IEEE International Conference on Condition Monitoring and Diagnosis 23-27 September 2012, Bali, Indonesia.

[4] IEC 61000-4-20 Electromagnetic Compatibility (EMC)- Part 4: Testing and Measurement techniques. Section 20: Emission and Immunity Testing in Transverse Electromagnetic (TEM) Waveguides. International Electrotechnical Commission Geneva, Switzerland, Draft 2 CD 61000-4-20, 2001

[5] IEC 60270, "High Voltage Test Techniques-Partial Discharge measurement," IEC, 2000-2012.

[6] P. G. Reddy, K. Prasanta, "Detection and Analysis of Partial Discharge using Ultra High Frequency Sensor" International Conference on Magnetics, Machines \& Drives, 24-26 July 2014, Kottayam, India.

[7] M. Zanjani, A. Akabari, H.R, N. Shhirdel,“ Investigating Partial Discharge UHF Electromagnetic Waves Propagation in Transformers Using FDTD Technique and 3D Simulation," IEEE International Conference on Condition Monitoring and Diagnosis 23-27 September 2012, Bali, Indonesia

[8] C. Sebastian, T. Stefan, "Location of PD Sources in Power Transformers by UHF and Acoustic Measurements, " IEEE International Conference on Dielectric and Electrical Insulation, December 2012 - Vol. 19, Issue. 6, pp.1934-1940

[9] M. D. Judd, L. Yang, I.B.B. Hunter, "Partial Discharge Monitoring for Power Transformers Using UHF Sensors Part 2: Field Experience," IEEE Electrical Insulation Magazine, IEEE, June 2005-Vol. 3, Issue. 3, pp.5-13.

[10] D. H. Froula, S. H. Glenzer, N. C. Luhmann, Jr., and J. Sheffield, Plasma Scattering of Electromagnetic Radiation, 2nd ed., Elsevier, 2001. 
[11] Martin D. Judd, Li Yang, Ian B. B. Hunter, "Partial Discharge Monitoring for Power Transformers Using UHF Sensors Part 2: Field Experience", IEEE Electrical Insulation Magazine, May/June 2005 Vol. 21, No. 3

[12] M. D. Judd, G. P. Cleary, C. J. Bennoch, Pearson, and J.S Pearson, and $\mathrm{T}$. Breckenridge, "Power transformer monitoring using UHF sensors: site trials; Electrical Insulation", Conference Record of the IEEE International Symposium on Electrical Insulation, Boston, USA, pp, $145-149,2002$.

[13] Z. Xu, C. Yonghong, M. Yongpeng, W. Kai, N. Yuhan, "The propagation characteristics of UHF partial discharge in power transformers with complex windings structure, " 2012 Annual Report Conference on Electrical Insulation and Dielectric Phenomena (CEIDP). Montreal, QC, Canada, Tech. Report. 13174840, 14-17 Oct. 2012.
[14] M. Joko., UHF Bowtie Antenna Modeling and Enhancement as GAS Insulated Substation (GIS) Partial Discharge (PD) Sensor, M. S. thesis, Bandung Institute of Technology, 2013.

[15] A. TAFLOVE. "Computational Electrodynamics: The FiniteDifference Time-Domain Method" 3nd ed., Boston, London, 2005, pp. 229-267.

[16] K. S. Yee., Numerical solution of Initial Boundary Value Problems Involving Maxell's Equations in Isotropic Media, In IEEE Transaction on Antenna and Propagation, vol. 14.No.3, May 1996,pp.302-307.

[17] J. P. Berenger, "Three-Dimensional Perfectly Matched Layer for the Absorption of Electromagnetic Waves", Journal of Computational Physics, September 1996- Vol. 127, Issue. 2, pp.363-379.

[18] W. Li, S. Su and L. Zhou, "The Deduction of Partial Discharge Pulse Current from Its Radiating UHF Signal," IEEE Power Engineering Conference, IPEC, pp. 193-198, Jan. 2005. 